\title{
RAPID COMPUTATION OF OCCULTATION LIGHTCURVES USING FOURIER DECOMPOSITION
}

\author{
LESLIE A. Young \\ Southwest Research Institute, 1050 Walnut St, Boulder, CO 80302, USA; layoung@boulder.swri.edu \\ Received 2008 October 11; accepted 2008 November 17; published 2009 January 29
}

\begin{abstract}
Calculation of a stellar occultation lightcurve from an assumed refractivity profile involves the integral of the refractivity or its derivatives along the line of sight through an atmosphere. For the general case, normal numerical integration can be time consuming for least-squares fitting with a modest number of free parameters, or for calculation at a fine grid for comparison with local refocusing ("spikes") in a lightcurve. A new method, based on the Fourier decomposition of the refractivity profile, can rapidly calculate the line-of-sight integrals needed for occultations. The method is formulated for small planets, to be applicable to Pluto and Triton. The Fourier decomposition method may have applicability to a wider range of atmospheric studies.
\end{abstract}

Key words: methods: numerical - occultations

Online-only material: color figure

\section{INTRODUCTION}

With the passage of Pluto into the Galactic plane, there have been a recent influx of extremely high-quality occultation lightcurves (e.g., Pasachoff et al. 2005, Young et al. 2008), allowing an investigation of Pluto's atmospheric structure as a function of time, latitude, and local time of day. While the relationship between the atmospheric structure and the occultation lightcurve has been solved for the case of temperatures proportional to powers of the radius (Elliot \& Young 1992; Eshleman \& Gurrola 1993), the temperature profiles actually encountered in atmospheres are more complicated. Furthermore, the quality of the recent Pluto occultations can support least-squares fitting to a larger number of free parameters. Chamberlain \& Elliot (1997) present a method of calculating a grid of occultation lightcurves from arbitrary atmospheric models. While this improved computation speed, direct integration can still be prohibitively slow for fitting models complex enough to fully interpret these recent data.

A second problem in occultation studies that is limited by computation speed is the study of the characteristics of the smallest scale structures in dense planetary atmospheres, usually referred to as spikes. These are caused by temperature fluctuations that appear to be similar in character to saturated gravity waves seen in the Earth's atmosphere (McLandress 1988). Of particular interest are the power spectrum of the temperature fluctuations and the histogram of temperature gradients. Unfortunately, the power spectra of temperature fluctuations tend to be muted and distorted when derived from Abel transforms, which cannot account for ray crossing, wave optics, or the finite sizes of stars (Sicardy et al. 1999). Forward modeling can account for these effects explicitly. However, an extremely rapid method of calculating occultation lightcurves with forward modeling must be used to study waves at the smallest sizes.

Such a method of rapid computation of occultation lightcurves uses Fourier decomposition of the refractivity profile. The key to this method is the definition of the refractivity profile as the product of a baseline refractivity profile that is roughly exponential with radius and a scaling factor that is decomposed into its Fourier components. The exponential term from the baseline atmosphere can then be combined with the sine and cosine terms in the Fourier decomposition to form expressions with imaginary scale heights. This leads to expressions for the pressure, line-of-sight integral of refractivity, bending angle, and bending angle derivative based on simple manipulations of the Fourier decomposition of the refractivity. Section 2 reviews the general occultation equations. Section 3 presents the equations for the baseline profiles of refractivity, its lineof-sight integral, bending angle, bending angle derivative, and pressure, all in forms most useful to the subsequent application to the general refractivity profile. The heart of the method is demonstrated in Section 4, with detailed derivations for the line-of-sight integral of refractivity and for pressure. A simple example is presented in Section 5. The possible applications of the model are discussed in Section 6.

\section{GENERAL OCCULTATION EQUATIONS}

In this section, I present the equations relating the refractivity to other quantities, without derivation. The reader is referred to, e.g., Elliot \& Young (1992), Wasserman \& Veverka (1973), or Eshleman \& Gurrola (1993) for further background.

The pressure $(p)$ is related to the refractivity $(v)$ by

$$
p(r)=\int_{r}^{\infty} g\left(r^{\prime}\right) \mu\left(r^{\prime}\right) m_{\mathrm{amu}} \frac{L}{v_{\mathrm{STP}}\left(r^{\prime}\right)} v\left(r^{\prime}\right) d r^{\prime}
$$

where $r$ is the radius from the planet center, $r^{\prime}$ is the variable of integration, $g$ is the gravitational acceleration, $\mu$ is the molecular weight, $m_{\mathrm{amu}}$ is the weight of an atomic mass unit, $v_{\mathrm{STP}}$ is the refractivity at standard temperature and pressure, and $L$ is Loschmidt's number. Often, pressure is written in terms of the scale height $(H)$, as $d \ln p=-d r / H$. Here I write the pressure as an integral of refractivity for use with the Fourier decomposition of refractivity later. For constant atmospheric composition, $\mu$ and $\nu_{\text {STP }}$ are independent of $r$. However, since Earth-based stellar occultations typically probe near the homopause of the occulting atmosphere, I write the pressure in the general form.

I derive the temperature $(T)$ using the ideal gas law, so that

$$
T(r)=\frac{p(r) \nu_{\mathrm{STP}}(r)}{k L v(r)} .
$$

The line-of-sight integral of refractivity $(\alpha)$ is given by

$$
\alpha(r)=2 \int_{0}^{\infty} v\left(r^{\prime}\right) \frac{r^{\prime}}{\sqrt{r^{\prime 2}-r^{2}}} d r^{\prime},
$$


Table 1

Fourier Transforms of Occultation Parameters

\begin{tabular}{|c|c|c|c|c|c|c|}
\hline Quantity & $f_{L}$ & $f_{1}$ & $f_{2}$ & $f_{3}$ & $f_{4}$ & $\beta$ \\
\hline$v$ & $\bar{v}(z)$ & 0 & 0 & 0 & 0 & 0 \\
\hline$\alpha$ & $\bar{v}(z) r \sqrt{2 \pi \delta}$ & $\frac{9-b}{8}$ & $\frac{345+46 b-7 b^{2}}{128}$ & $\frac{9555+5455 b+452 b^{2}-75 b^{3}}{1024}$ & $\begin{array}{l}\frac{1371195}{32768}+\frac{386421}{8192} b \\
+\frac{251153}{16384} b^{2}+\frac{6741}{8192} b^{3}-\frac{5509}{32768} b^{4}\end{array}$ & $1 / 2$ \\
\hline$\theta$ & $-\bar{v}(z) \sqrt{\frac{2 \pi}{\delta}}$ & $\frac{-3+3 b}{8}$ & $\frac{-15+14 b+b^{2}}{128}$ & $\frac{-105+27 b+69 b^{2}+9 b^{3}}{1024}$ & $\begin{array}{l}-\frac{4725}{32768}-\frac{1059}{8192} b+ \\
\frac{2353}{16384} b^{2}+\frac{3764}{8192} b^{3}+\frac{491}{32768} b^{4}\end{array}$ & $-1 / 2$ \\
\hline$\frac{d \theta}{d r}$ & $\frac{\overline{\mathrm{v}}(z)}{r} \sqrt{\frac{2 \pi}{\delta^{3}}}$ & $\frac{1+15 b}{8}$ & $\frac{9-34 b+25 b^{2}}{128}$ & $\frac{75-81 b+b^{2}+5 b^{3}}{1024}$ & $\begin{array}{l}\frac{3675}{32768}-\frac{339}{8192} b \\
-\frac{10555}{1684} b^{2}-\frac{67}{8192} b^{3}+\frac{59}{32768} b^{4}\end{array}$ & $-3 / 2$ \\
\hline$p$ & $\bar{v}(r) g(r) \mu \mathrm{m}_{\mathrm{amu}} \frac{L}{v_{\mathrm{STP}}} r \delta$ & 0 & 0 & 0 & $\begin{array}{ccc}10384 & 0 & 52108\end{array}$ & 1 \\
\hline
\end{tabular}

and the bending angle $(\theta)$ is

$$
\theta(r)=\frac{d \alpha(r)}{d r}
$$

For planets with spherical symmetry, the bending angle and its derivative are needed to calculate an observed lightcurve. The radius in the shadow plane $(\rho)$ is

$$
\rho(r)=r+D \theta(r)
$$

where $D$ is the observer-atmosphere distance. The flux from a single location in the atmosphere, for a spherical atmosphere with no extinction, is

$$
\phi(r)=\left|\frac{1}{1+D \theta(r) / r}\right| \times\left|\frac{1}{1+D d \theta(r) / d r}\right| .
$$

\section{BASELINE ATMOSPHERE}

Throughout, I use the small-planet assumptions, where gravity varies as $r^{-2}$, and terms to first few orders in the ratio of scale height over radius are kept. This will allow this method to be used for Pluto, where the radius is only $\sim 20$ times larger than the scale height. The starting point of this formulation is the baseline refractivity $(\bar{v})$, where baseline quantities are indicated with an overbar, to distinguish them from the general atmosphere in Section 4. The intent is to describe an analytic refractivity profile that approximates the general profile over the region of interest. This requires a baseline refractivity that depends on three parameters - two to ensure a match between the baseline refractivity and the general profile at the ends of the region of interest, and one to control the curvature of the log of refractivity.

A suitable choice for a baseline atmosphere is one with constant composition and temperature that varies as $r^{\mathrm{b}}$. This model has two advantages. First, the temperature never reaches negative values, and second, this class of atmosphere has been previously studied in the context of occultations (Elliot \& Young 1992; Eshleman \& Gurrola 1993). With these assumptions, the refractivity over the region of interest can be described as

$$
\bar{v}(z)=v_{\text {ref }}\left(\frac{r}{r_{\text {ref }}}\right)^{-b} e^{-z / H_{\text {ref }}}
$$

where the pseudoaltitude $(z)$ is defined by

$$
z \equiv\left\{\begin{array}{ll}
\frac{r_{\text {ref }}}{1+b}\left[1-\left(\frac{r}{r_{\text {ref }}}\right)^{-(1+b)}\right], & b \neq-1 \\
r_{\text {ref }} \ln \left(r / r_{\text {ref }}\right) & b=-1
\end{array} .\right.
$$

where the subscript $r e f$ refers to conditions at a reference radius. Note that $z$ is a function only of radius $(r)$, the reference radius $\left(r_{\text {ref }}\right)$, and the temperature power $(b)$. In particular, $z$ does not depend on the scale height or temperature. For large planets, or for temperatures varying in proportion to gravity $(b=-2), z \approx$ $r-r_{\text {ref }}$ is the altitude above the reference radius. The ratio of the radius to the pressure scale height $(\lambda)$ at radius $r$ is given by

$$
\lambda=\left(\frac{r_{\text {ref }}}{H_{\text {ref }}}\right)\left(\frac{r}{r_{\text {ref }}}\right)^{-(1+b)} .
$$

for an atmosphere with constant composition and a refractivity profile given by Equation (7) (Elliot \& Young 1992). For $b>-1$, $z$ has a maximum value of $r_{\text {ref }} /(1+b)$, and $\alpha$ is infinite for $1>b \geqslant$ -1 (Eshleman \& Gurrola 1993). This is resolved physically by noting that the equations of hydrostatic equilibrium fail as $\lambda \approx$ 1, so that Equation (7) is valid only for radii below the exobase. It is resolved mathematically by integrating Equation (3) to the exobase, rather than infinity. For $b=-1$, where the functional form of the refractivity is a power law (Eshleman \& Gurrola 1993), I define $z$ in Equation (8) to allow the combination of a real scale height with imaginary arguments in the Fourier decomposition.

The line-of-sight integral of refractivity $(\alpha)$ is derived in the Appendix, using the same approach applied to the bending angle integral in Elliot \& Young (1992). To first order in the ratio of scale height to radius $(\delta=1 / \lambda)$, this is

$$
\bar{\alpha}(z)=\bar{v}(z) r \sqrt{2 \pi \delta}\left[1+\frac{9-b}{8} \delta+O\left(\delta^{2}\right)\right] .
$$

The baseline quantities can be written as a lead term $\left(f_{\mathrm{L}}\right)$ multiplied by a series in $\delta$.

$$
\bar{f}(z) \equiv f_{L}\left[1+f_{1} \delta+f_{2} \delta^{2}+f_{3} \delta^{3}+f_{4} \delta^{4}+\cdots\right] .
$$

The full coefficients to fourth order are given in Table 1. The corresponding leading terms and coefficients for baseline bending angle $(\bar{\theta})$, and bending angle derivative $(d \bar{\theta} / d r)$ are also given in Table 1, using the expansions from Elliot \& Young (1992).

The pressure can be derived from the refractivity either by the application of the ideal gas law or by the integral in Equation (1). Starting with Equation (1) makes explicit how the pressure depends on the scale height, for use with the complex scale height in Section 4. For gravity proportional to $r^{-2}$ and constant composition, the pressure becomes

$$
\bar{p}(r)=\bar{v}(r) g(r) \mu \mathrm{m}_{\mathrm{amu}} \frac{L}{v_{\mathrm{STP}}} \int_{r}^{\infty}\left(r^{\prime} / r\right)^{-(2+b)} e^{-\left(z^{\prime}-z\right) / H_{\mathrm{ref}}} d r^{\prime}
$$


where $z^{\prime}$ is defined by Equation (8), substituting $r^{\prime}$ for $r$. Performing the integral gives

$$
\bar{p}(r)=\bar{v}(r) g(r) \mu \mathrm{m}_{\mathrm{amu}} \frac{L}{v_{\mathrm{STP}}} r \delta .
$$

The baseline temperature $(\bar{T})$ is given by the ideal gas law, Equation (2).

$$
\bar{T}(r)=\frac{g(r) \mu \mathrm{m}_{\mathrm{amu}}}{k} r \delta .
$$

\section{FOURIER DECOMPOSITION}

The refractivity can be generalized for nonconstant scale heights by writing the refractivity as the product of a scale factor $\left(\sigma_{v}\right)$ and the baseline refractivity:

$$
v(z)=\sigma_{\nu}(z) \bar{v}(z)=\sigma_{\nu}(z) v_{\text {ref }}\left(r / r_{\text {ref }}\right)^{-b} e^{-z / H_{\text {ref }}} .
$$

I express the scale factor as a Fourier transform in vertical wavenumber $(m)$. I introduce the operators $F$ and $F^{-1}$ as the forward and inverse Fourier transforms, so that

$$
\begin{array}{r}
\hat{\sigma}_{\nu}(m)=F\left\{\sigma_{\nu}\right\}(m):=\int_{-\infty}^{\infty} e^{-i m z} \sigma_{\nu}(z) d z \\
\sigma_{\nu}(z)=F^{-1}\left\{\hat{\sigma}_{\nu}\right\}(z):=\frac{1}{2 \pi} \int_{-\infty}^{\infty} e^{i m z} \hat{\sigma}_{\nu}(m) d m .
\end{array}
$$

Substituting Equations (15) and (17) into Equation (3) yields

$$
\begin{aligned}
\alpha(z)= & 2 \int_{0}^{\infty} v_{\text {ref }}\left(r^{\prime} / r_{\text {ref }}\right)^{-b} e^{-z^{\prime} / H_{\text {ref }}}\left\{\frac{1}{2 \pi} \int_{-\infty}^{\infty} e^{i m z^{\prime}} \hat{\sigma}_{\nu}(m) d m\right\} \\
& \times \frac{r^{\prime}}{\sqrt{r^{\prime 2}-r^{2}}} d r^{\prime} .
\end{aligned}
$$

I then define a complex scale height $H_{m}$

$$
H_{m}=\frac{H_{\mathrm{ref}}}{1-i m H_{\mathrm{ref}}}
$$

so that

$$
-\frac{z^{\prime}}{H_{\text {ref }}}+i m z^{\prime}=-\frac{z^{\prime}}{H_{m}}
$$

and reverse the order of integration

$$
\begin{aligned}
\alpha(z)= & \frac{1}{2 \pi} \int_{-\infty}^{\infty} \hat{\sigma}_{\nu}(m) \\
& \times\left\{2 \int_{0}^{\infty} v_{\mathrm{ref}}\left(r^{\prime} / r_{\mathrm{ref}}\right)^{-b} e^{-z^{\prime} / H_{m}} \frac{r^{\prime}}{\sqrt{r^{\prime 2}-r^{2}}} d r^{\prime}\right\} d m .
\end{aligned}
$$

Following Equation (10), this becomes

$$
\begin{aligned}
\alpha(z)= & \frac{1}{2 \pi} \int_{-\infty}^{\infty} \hat{\sigma}_{\nu}(m)\left\{\sqrt{2 \pi \delta_{m}} r v_{\mathrm{ref}}\left(r / r_{\mathrm{ref}}\right)^{-b} e^{-z / H_{m}}\right. \\
& \left.\times\left[1+\frac{9-b}{8} \delta_{m}+O\left(\delta_{m}^{2}\right)\right]\right\} d m
\end{aligned}
$$

where

$$
\delta_{m}=\left(\frac{H_{m}}{r_{\text {ref }}}\right)\left(\frac{r}{r_{\text {ref }}}\right)^{1+b}=\frac{H_{m}}{H_{\text {ref }}} \delta .
$$

By writing $\exp \left(-z / H_{m}\right)=\exp \left(-z / H_{\text {ref }}\right) \exp (i m z)$ and pulling out of the integral all terms that are independent of $m$, this can be written as the following sum:

$$
\begin{aligned}
\alpha(z)= & \bar{v}(z) r \sqrt{2 \pi \delta}\left[\frac{1}{2 \pi} \int_{-\infty}^{\infty} \hat{\sigma}_{v}(m)\left\{\sqrt{\frac{H_{m}}{H_{r e f}}} e^{-z / H_{m}}\right\} d m\right. \\
& \left.+\left[\frac{9-b}{8} \delta\right] \frac{1}{2 \pi} \int_{-\infty}^{\infty} \hat{\sigma}_{\nu}(m)\left\{\sqrt{\frac{H_{m}}{H_{\mathrm{ref}}}} e^{-z / H_{m}} \frac{H_{m}}{H_{\mathrm{ref}}}\right\} d m\right] \\
& +O\left(\delta_{m}^{2}\right) .
\end{aligned}
$$

At this point, it is convenient to define the following function,

$$
S_{\beta}(z)=\left[F^{-1}\left\{\hat{\sigma}_{v}(m)\left(H_{m} / H_{\text {ref }}\right)^{\beta}\right\}(z)\right],
$$

with which Equation (24) becomes

$$
\alpha(z)=\bar{v}(z) r \sqrt{2 \pi \delta}\left(S_{1 / 2}(z)+\frac{9-b}{8} \delta S_{3 / 2}(z)+O\left(\delta^{2}\right)\right)
$$

The pressure integral proceeds similarly. Substituting Equations (15) and (17) into Equation (1) gives

$$
\begin{aligned}
p(r)= & \int_{r}^{\infty} g\left(r^{\prime}\right) \mu\left(r^{\prime}\right) m_{\mathrm{amu}} \frac{L}{v_{\mathrm{STP}}\left(r^{\prime}\right)} v_{\mathrm{ref}}\left(r^{\prime} / r_{\mathrm{ref}}\right)^{-b} e^{-z^{\prime} / H_{\mathrm{ref}}} \\
& \times\left\{\frac{1}{2 \pi} \int_{-\infty}^{\infty} e^{i m z^{\prime}} \hat{\sigma}_{\nu}(m) d m\right\} d r^{\prime}
\end{aligned}
$$

Reversing the order of integration gives

$$
\begin{aligned}
p(r)= & \frac{1}{2 \pi} \int_{-\infty}^{\infty} \hat{\sigma}_{v}(m)\left\{\int_{r}^{\infty} g\left(r^{\prime}\right) \mu\left(r^{\prime}\right) m_{\mathrm{amu}}\right. \\
& \left.\times \frac{L}{v_{\mathrm{STP}}\left(r^{\prime}\right)} v_{\mathrm{ref}}\left(r^{\prime} / r_{\mathrm{ref}}\right)^{-b} e^{-z^{\prime} / H_{\mathrm{ref}}} e^{i m z^{\prime}} d r^{\prime}\right\} d m
\end{aligned}
$$

The inner integral is solved with Equation (13), giving

$$
p(r)=\frac{1}{2 \pi} \int_{-\infty}^{\infty} \hat{\sigma}_{\nu}(m) \bar{v}(r) g(r) \mu m_{\mathrm{amu}} \frac{L}{v_{\mathrm{STP}}} r \delta_{m} e^{i m z} d m .
$$

As with the $\alpha$ decomposition, factoring out terms that depend on $r$ allows pressure to be expressed in terms of Fourier transforms

$$
p(r)=\bar{v}(r) g(r) \mu m_{\mathrm{amu}} \frac{L}{v_{\mathrm{STP}}} r \delta \frac{1}{2 \pi} \int_{-\infty}^{\infty} \hat{\sigma}_{v}(m) \frac{H_{m}}{H_{\mathrm{ref}}} e^{i m z^{\prime}} d m,
$$

expressed simply as

$$
p(r)=\bar{v}(r) g(r) \mu m_{\mathrm{amu}} \frac{L}{v_{\mathrm{STP}}} r \delta S_{1}(z)
$$

The bending angle and bending angle derivative also involve only the linear operators of integration and differentiation, allowing a similar manipulation of the decomposed refractivity. 


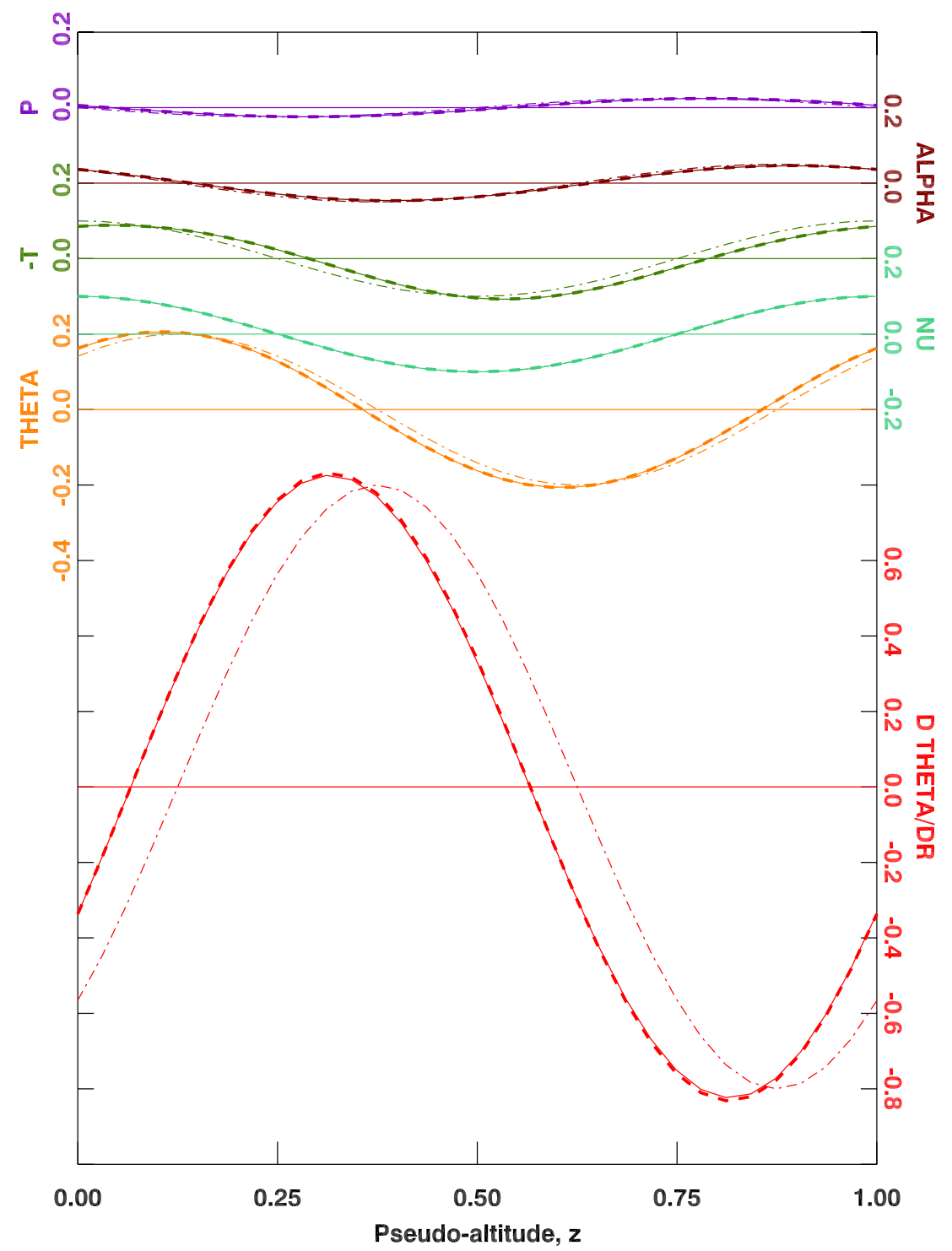

Figure 1. Plot of the perturbation terms of temperature $(T)$, pressure $(p)$, refractivity $(v)$, line-of-sight integral of refractivity $(\alpha)$, bending angle $(\theta)$, and bending angle derivative $(d \theta / d r)$. Refractivity was calculated with $b=0, \varepsilon_{v}=0.1, m=2 \pi, H_{\text {ref }}=4 / m$, and $r_{\text {ref }}=20 H_{\text {ref }}$ (see the text). Solid lines show the calculation using traditional line-of-sight integrals as in Chamberlain \& Elliot (1997). Thick dashed lines show the calculation with the Fourier decomposition method presented here, calculated to first order in the ratio of the scale height to radius, showing good agreement with direct integration. Thin dot-dashed lines plot the approximate wave relations, showing the relative amplitudes and phase shifts of the various atmospheric quantities.

(A color version of this figure is available in the online journal.)

To first order in $\delta$, these are

$$
\begin{aligned}
& \theta(z)=-\bar{v}(z) \sqrt{2 \pi / \delta}\left(S_{-1 / 2}(z)-\frac{3-3 b}{8} \delta S_{1 / 2}(z)+O\left(\delta^{2}\right)\right) \\
& \frac{d \theta(z)}{d r}=\frac{\bar{v}(z)}{r} \sqrt{\frac{2 \pi}{\delta^{3}}}\left(S_{-3 / 2}(z)+\frac{1+15 b}{8} \delta S_{-1 / 2}(z)+O\left(\delta^{2}\right)\right) .
\end{aligned}
$$

In analogy to Equation (11), the pressure, line-of-sight integral, bending angle, and bending angle derivative can be written to higher order in $\delta$ as

$$
\begin{aligned}
f(z) \equiv & f_{L}\left[S_{\beta}+f_{1} S_{\beta+1} \delta+f_{2} S_{\beta+2} \delta^{2}\right. \\
& \left.+f_{3} S_{\beta+3} \delta^{3}+f_{4} S_{\beta+4} \delta^{4}+\cdots\right]
\end{aligned}
$$

where $\beta$ is the power of $\delta$ in the leading term, given in Table 1 .

\section{EXAMPLE: ISOTHERMAL ATMOSPHERE WITH A SINGLE WAVE}

I tested this method numerically with an example of a baseline atmosphere with $b=0, H_{\text {ref }}=2 / \pi$, and $r_{\text {ref }}=40 / \pi$, where $H_{\text {ref }}$ and $r_{\text {ref }}$ are in arbitrary units. I then added a single wave to the baseline refractivity, $\sigma_{v}(z)=1+\varepsilon_{\nu} \cos \left(m_{0} z\right)$, with $\varepsilon_{v}=$ $0.1, m_{0}=2 \pi$. This combination gives $r_{\text {ref }} / H_{\text {ref }}=20$, so that small-planet effects cannot be ignored, and $H_{\text {ref }} m_{0}=4$, so the wavelength is small compared with the scale height. The choice of $m_{0}=2 \pi$ gives a vertical wavelength $\left(L_{z}=2 \pi / m_{0}\right)$ of 1 . The refractivity was calculated on an evenly spaced grid of pseudoaltitude $z$, with 32 points per wavelength, spanning $z=-5.5$ to $z=19.6$.

The Fourier method was tested against previously written and tested benchmark code that calculates the line-of-sight integral for an arbitrary grid of function values and radii. This routine treats successive pairs of radii as the bounds of an 
atmospheric shell. Within each shell, the integrand is treated as an exponential with a single scale height. For each shell, the minimum and maximum value of the distance along the line of sight $\left(x=\left(r^{2}-r^{3}\right)^{1 / 2}\right)$ is found. The function is evaluated at 20 points per shell, evenly spaced in $x$. The perturbation term $\left(\varepsilon_{f}=\left(f-f^{\prime}\right) / f^{\prime}\right)$ found by the benchmark calculation is shown as thin, solid lines in Figure 1. The perturbation term calculated by the method outlined in this paper is plotted with thick dashed lines in Figure 1, showing the good agreement between the two methods.

It is useful to consider the effect on a single wave with wavenumber $m_{0}$ where $m_{0} H_{\text {ref }} \gg 1$, so that

$$
\frac{H_{m}}{H_{\text {ref }}} \approx \frac{i}{m_{0} H_{\text {ref }}} .
$$

In this case, the Fourier transform of $v$ is only non-zero at wavenumbers $-m_{0}, 0$, or $m_{0}$

$$
\hat{\sigma}_{v}=\pi \varepsilon_{v}\left(\delta_{\text {dirac }}\left(-m_{0}\right)+\delta_{\text {dirac }}\left(m_{0}\right)\right)+2 \pi \delta_{\text {dirac }}(0)
$$

where $\delta_{\text {dirac }}$ is the Dirac delta function (not to be confused with the ratio of scale height to radius, $\delta$ ).

Application of Equation (25) yields

$$
S_{\beta}(z)=\varepsilon_{v}\left(m_{0} H_{\mathrm{ref}}\right)^{-\beta} \cos \left[m_{0}\left(z+L_{z} \beta / 4\right)\right]+1 .
$$

In the limit of large-planets $(\delta \ll 1)$ and short wavelengths $\left(m_{0} H_{\text {ref }} \gg 1\right)$, the application of Equation (37) shows that the amplitudes of the scaled perturbations of $p$ and $\alpha$ are smaller than for $v$ (Equations (38(a)), (38(b))), and shifted to smaller $r$, while the opposite is the case for $\theta$ and $d \theta / d r$ (Equations 38(c), $38(d))$. Because the pressure perturbations are small compared to the refractivity (or density), the temperature perturbation is approximately equal in magnitude to the refractivity (or density) perturbation, but opposite in sign (Equation 38(e)):

$$
\begin{gathered}
\varepsilon_{p} \approx \varepsilon_{v}\left(m_{0} H_{\mathrm{ref}}\right)^{-1} \cos \left[m_{0}\left(z+L_{z} / 4\right)\right] \\
\varepsilon_{\alpha} \approx \varepsilon_{v}\left(m_{0} H_{\mathrm{ref}}\right)^{-1 / 2} \cos \left[m_{0}\left(z+L_{z} / 8\right)\right] \\
\varepsilon_{\theta} \approx \varepsilon_{v}\left(m_{0} H_{\mathrm{ref}}\right)^{1 / 2} \cos \left[m_{0}\left(z-L_{z} / 8\right)\right] \\
\varepsilon_{d \theta / d r}=\varepsilon_{v}\left(m_{0} H_{\mathrm{ref}}\right)^{3 / 2} \cos \left[m_{0}\left(z-3 L_{z} / 8\right)\right] \\
\varepsilon_{T}=-\varepsilon_{v} \cos \left[m_{0} z\right] .
\end{gathered}
$$

The approximate wave relations are plotted as thin dot-dashed lines in Figure 1. These demonstrate the relative magnitudes and phase shifts. The difference between the approximate relations and the perturbations calculated by direct integration or Fourier decomposition is due to the small but non-negligible sizes of $\delta$ and $1 /\left(m_{0} H_{\text {ref }}\right)$.

\section{DISCUSSION}

The method was motivated by the two problems raised in the introduction: the need to fit more complex thermal models to recent Pluto stellar occultations, and the need for a forward modeling approach for the study of small-scale structure indicated by spikes in stellar occultations of the jovian planets and Titan. However, the method laid out in this paper is very general, and decomposition of an atmospheric profile may have utility for a larger range of problems.

The approximate wave relations can be used to quickly derive at what radii waves of a given wavelength and amplitude will violate the assumptions of the Abel inversion of stellar occultations lightcurves.

Wavelet analysis can be easily done by noting that a wavelet is often expressed compactly in the spectral domain. The relationship derived here between the Fourier components of various atmospheric quantities means that the spectral components of a wavelet in $\theta$ or $d \theta / d r$ can be written down directly, given the spectral components in the corresponding wavelet in refractivity.

Because the phase delay is proportional to the line-ofsight integral of refractivity, this method is applicable to radio occultations as well as stellar, with the caveat that the source's path through the atmosphere is straight.

The equations for $\alpha$ can be easily modified to calculate the line-of-sight integral of absorption, making this method useful for UV occultations. Having a rapid method of calculating UV stellar occultation spectral light curves will allow a more thorough study of the sensitivity of derived temperatures to the upper boundary conditions.

Finally, the approach outlined here can be applied to any linear operator acting on a roughly exponential function of radius, such as atmospheric absorption viewed at a range of emission angles.

This research was initially motivated by Uranian occultation data provided by Richard French, who also contributed to the original, large-planet formulation of this method. Discussions with Edward Dunham helped with make this applicable to small planets. The flow of this paper was greatly improved by discussions with Eliot Young, Marc Buie, and Catherine Olkin. This research was supported, in part, by NASA grant NNG05GF05G,

\section{APPENDIX}

\section{SERIES FORM OF THE LINE-OF-SIGHT INTEGRAL OF REFRACTIVITY}

The derivation of the series approximation to the line-of-sight integral of refractivity $(\alpha)$ follows that of Elliot \& Young 1992 (EY92). For this Appendix, I use the terminology from EY92, which differs slightly from the terminology used in the body of the paper.

Given temperature and molecular weight profiles written as

$$
\mu=\mu_{0}\left(r / r_{0}\right)^{-a}
$$

and

$$
T=T_{0}\left(r / r_{0}\right)^{b}
$$

from which $\lambda_{\mathrm{g}}$, the ratio of radius to scale height, is

$$
\lambda_{\mathrm{g}}(r)=\lambda_{\mathrm{g} 0}\left(r / r_{0}\right)^{-(1+a+b)}
$$

and the refractivity is

$$
v(r)=v_{0}\left(\frac{r}{r_{0}}\right)^{-b} \exp \left(\frac{\lambda_{\mathrm{g}}(r)-\lambda_{\mathrm{g} 0}}{1+a+b}\right) .
$$

I use the same geometry as in EY92, where $x$ is the coordinate that lies along the path of the ray and has its origin at the closest 
approach of the ray to the center of the planet. If $r$ is the radius at closest approach, and $r^{\prime}$ is the radius at $x$, then

$$
r^{\prime 2}=x^{2}+r^{2}
$$

The line-of-sight integral of refractivity is

$\alpha(z)=\int_{-\infty}^{\infty} v\left(r^{\prime}\right) d x=v(r) \int_{-\infty}^{\infty}\left(\frac{r}{r^{\prime}}\right)^{b} \exp \left[\frac{\lambda\left(r^{\prime}\right)-\lambda(r)}{1+a+b}\right] d x$

I make the same substitution as in $\operatorname{EY92}\left(\delta \equiv 1 / \lambda_{\mathrm{g}}(r)\right)$ and define the same variable of integration, $y$,

$$
y=(x / r) \sqrt{1 /(2 \delta)} .
$$

Equation (A6), expressed in terms of $y$, is

$$
\begin{aligned}
\alpha(z)= & v(r) \sqrt{2 \delta} r \int_{-\infty}^{\infty}\left(1+2 \delta y^{2}\right)^{-b / 2} \\
& \times \exp \left[\frac{\left(1+2 \delta y^{2}\right)^{-(1+a+b) / 2}-1}{(1+a+b) \delta}\right] d y .
\end{aligned}
$$

Expanding the integrand in a series in $\delta$ gives

$$
\begin{aligned}
\alpha(z)= & v(r) \sqrt{2 \delta} r \int_{-\infty}^{\infty} e^{-y^{2}} \\
& \times\left\{1-\left[b y^{2}+\left(\frac{3+a+b}{2}\right) y^{4}\right] \delta\right. \\
& \left.+O\left(\delta^{2}\right)\right\} d y
\end{aligned}
$$

Using

$$
\int_{-\infty}^{\infty} y^{n} e^{-y^{2}} d x=\left\{\begin{array}{cc}
\Gamma((n+1) / 2), & n \text { even } \\
0 & n \text { odd }
\end{array}\right.
$$

and integrating term by term, one gets

$$
\alpha(z)=v(r) \sqrt{2 \delta} r A_{\alpha}(\delta, a, b)
$$

where

$$
\begin{aligned}
& A_{\alpha}(\delta, a, b)=1+\left(\frac{9+3 a}{8}-\frac{b}{8}\right) \delta \\
& +\left(\frac{345+310 a+65 a^{2}}{128}+\frac{23+5 a}{64} b-\frac{7 b^{2}}{128}\right) \delta^{2} \\
& +\left(\frac{9555+15155 a+7665 a^{2}+1225 a^{3}}{1024}\right. \\
& +\frac{5455+4970 a+1015 a^{2}}{\left.1024+\frac{452+35 a}{1024} b^{2}-\frac{75}{1024} b^{3}\right) \delta^{3}} \\
& +\left(\frac{21\left(65295+153204 a+130666 a^{2}+47764 a^{3}+6271 a^{4}\right)}{32768}\right. \\
& +\frac{21\left(18401+29993 a+15482 a^{2}+2491 a^{3}\right)}{8192} b \\
& +\frac{7\left(35879+32778 a+6399 a^{2}\right)}{16384} b^{2}+\frac{21(321-29 a)}{8192} b^{3} \\
& \left.-\frac{5509}{32768} b^{4}\right) \delta^{4}+\cdots
\end{aligned}
$$

\section{REFERENCES}

Chamberlain, D. M., \& Elliot, J. L. 1997, PASP, 109, 1170

Elliot, J. L., \& Young, L. A. 1992, AJ, 103, 3

Eshleman, V. R., \& Gurrola, E. M. 1993, Icarus, 105, 298

McLandress, C. 1988, JASTP, 60, 1357

Pasachoff, J. M., et al. 2005, AJ, 129, 1718

Sicardy, B., et al. 1999, Icarus, 142, 357

Wasserman, L. H., \& Veverka, J. 1973, Icarus, 20, 322

Young, E. F., et al. 2008, AJ, 136, 1757 